\title{
Tabaquismo en el adolescente
}

\author{
ANA MARÍA HERRERA G.* y MARÍA PAZ CORVALÁN B.**
}

\section{Adolescents and smoking}

Smoking in adolescents produces addiction to nicotine, damage to the cardiorespiratory apparatus, and it is also a gateway to the use of illicit drugs. In the United States, electronic cigarettes are more frequently used by young people than conventional cigarettes, believing that they are less harmful to health, however, it is known that these devices produce combustion and deliver nicotine inhaled so their use leads traditional cigarette consumption and exposure to carcinogenic substances. For all of the above, tobacco control efforts must be: public policies (tobacco tax increases, mass advertising campaigns, smoke-free environments, restriction of advertising), early register and timely treatment of adolescents who have initiated tobacco consumption through the ABC-D as it appears in the First Guidelines of Clinical Practice of Smoking Treatment, Chile 2017, for this it is necessary that the health professionals receive training. Regarding second-hand smoke, pediatricians must refer parents to smoking cessation that can be telephone (6003607777, Salud Responde MINSAL, this number appears on cigarette packets).

Key words: Smoking; smoking cessation; tobacco use disorders; adolescents; public policy.

\section{Resumen}

El tabaquismo en adolescentes produce adicción a la nicotina, daño en el aparato cardiorrespiratorio, siendo además puerta de entrada para el uso de drogas ilícitas. En Estados Unidos, los cigarrillos electrónicos son más frecuentemente utilizados por los jóvenes que el cigarrillo convencional, creyendo que son menos dañinos para la salud, sin embargo, se sabe que estos dispositivos producen combustión y entregan nicotina por vía inhalada por lo que su uso lleva al consumo de cigarrillo tradicional y a la exposición a sustancias cancerígenas. Por todo lo anterior, los esfuerzos del control del tabaco deben estar puestos: en políticas públicas (aumento del impuesto al tabaco, campañas publicitarias masivas, ambientes cerrados libres de humo, restricción de la publicidad), la pesquisa precoz y tratamiento oportuno del adolescente que ya se ha iniciado en el consumo de tabaco a través del ABC-D tal como aparece en las Primeras Guías de Práctica Clínica de Tratamiento del Tabaquismo, Chile, 2017 para esto se necesita que los profesionales de salud reciban entrenamiento. Respecto al humo de segunda mano los pediatras deben derivar a los padres a cesación del tabaco que puede ser telefónica (6003607777, Salud responde MINSAL, este número aparece en las cajetillas de cigarrillos).

Palabras clave: Fumar; dejar de fumar; tabaquismo; adolescentes; políticas públicas.

En Chile, de acuerdo con el Décimo Estudio Nacional de Drogas realizado en más de 58.000 escolares de octavo básico a cuarto medio, se observó que la edad de inicio de la adicción era de 13,6 años en ambos sexos ${ }^{1}$. La tendencia de la serie de estudios del SENDA ${ }^{1}$ en población escolar chilena, muestra una sistemática reducción de la prevalencia de consumo en el último mes

* Dpto. Enfermedades Respiratorias Pediátricas. Clínica Santa María.

Facultad de Medicina de la Universidad de Los Andes.

Coordinadora Comisión Asma Infantil, Sociedad Chilena de Enfermedades Respiratorias.

** Programa de Tabaquismo Centro Médico Fundación del Banco Estado.

Coordinadora Comisión Tabaco, Sociedad Chilena de Enfermedades Respiratorias. 
(desde 51,6\% a 40\% entre los años 2001-2015) y por último la Encuesta Mundial de Tabaquismo en Jóvenes, EMTJ ${ }^{2}$, Chile 2016, lanzada recientemente, evidencia un cambio significativo (desde $34,2 \%$ a $17,2 \%$ entre los años 2008 y 2016 ) en prevalencia de fumadores actuales de cigarrillos en la población de 13 a 15 años de la Región Metropolitana (fumaron cigarrillo en algún momento de los últimos 30 días), también hubo disminución significativa en el consumo en el resto de las regiones evaluadas en este estudio: Arica y Parinacota, Tarapacá, Valparaíso y Bío- Bío. Si bien existen cambios importantes, aun es necesario bajar el consumo ${ }^{2}$. Dado que prácticamente el $100 \%$ del tabaquismo se inicia en la niñez y adolescencia, este grupo etario se ha convertido en la población objetivo de la industria que gasta billones de dólares al año en el marketing para sus productos, transmitiendo mensajes que hacen atractivo y común el uso del tabaco entre los jóvenes ${ }^{3}$. Dado que la nicotina hace que el humo sea áspero y difícil de fumar otra estrategia utilizada ha sido el uso de aditivos químicos que mejoran el sabor y dan suavidad ${ }^{3}$ Uno de ellos es el mentol, que refresca y adormece la garganta para reducir su irritación y hacer que el humo se sienta más suave ${ }^{4,5}$. Aparte del uso de aditivos, se ha aumentado el poder adictivo de los cigarrillos mediante el incremento de los niveles de nicotina $^{6}$. Otros factores muy importantes para iniciar el consumo de tabaco son: la influencia de los pares, el mal rendimiento escolar y el menor nivel de recursos económicos ${ }^{3}$. Se sabe que el uso temprano del tabaco trae inmediatamente serias consecuencias para la salud tales como adicción a la nicotina, efectos deletéreos en el aparato respiratorio y cardiovascular, siendo además un factor predisponente para el uso de drogas ilícitas ${ }^{3}$.

En las últimas décadas en Estados Unidos se ha observado una reducción del uso del cigarrillo convencional entre los jóvenes, sin embargo, de acuerdo a un informe reciente del Center of Disease Control and Prevention (CDC), hubo un dramático aumento del uso del cigarrillo electrónico en esta misma población ${ }^{7}$. En ese país la prevalencia de uso del cigarrillo electrónico en los últimos 30 días incrementó desde menos de un $2 \%$ en el año 2011 a cerca de un $11 \%$ en el 2015. Al respecto se pudo establecer que, en la actualidad en Estados Unidos, los cigarrillos electrónicos son más frecuentemente utilizados por los jóvenes que el cigarrillo convencional ${ }^{7}$. Una de las razones que dan los adolescentes para preferirlos es la creencia de que son menos dañinos para la salud, sin embargo se sabe que estos dispositivos entregan nicotina por vía inhalada, lo que produce adicción (es decir son puerta de entrada al consumo de cigarrillo tradicional), y además existe combustión lo que produce sustancias cancerígenas ${ }^{7}$. Por otro lado, la ingestión del líquido que contienen estos dispositivos puede causar toxicidad e incluso la muerte. Otras razones dadas por los jóvenes para el uso de cigarrillos electrónicos son la curiosidad por saber cómo son y el gusto por los saborizantes que contienen. La industria que promueve el uso del cigarrillo electrónico utiliza las mismas estrategias de venta que la industria del cigarrillo convencional y se ha visto que el gasto en marketing del cigarrillo electrónico continúa creciendo estimándose en 3,5 billones de dólares el año $2015^{7}$. Al ir incrementándose las ventas de cigarrillo electrónico ha ido disminuyendo paulatinamente el precio de estos dispositivos haciéndolos más asequibles a la población más joven. Si bien es cierto en nuestro país el uso de cigarrillo electrónico es poco frecuente en comparación con el uso del cigarrillo convencional, estos dispositivos ya están disponibles en el mercado nacional. El cigarrillo electrónico en Chile debe seguir la regulación que exige el ISP para la venta de cualquier fármaco, sin embargo ningún cigarrillo electrónico de los que se venden hasta la fecha ha pasado por el ISP, además el cigarrillo electrónico no está incluido en la actual legislación de control del tabaco. Todo lo anterior puede ser a futuro un problema de salud pública en nuestro país, sobre todo en la población adolescente deseosa de experimentar y al mismo tiempo vulnerable al manejo mediático.

Por todo lo expuesto, para evitar la iniciación de esta adicción, los esfuerzos deben estar puestos en políticas públicas (aumento del impuesto al tabaco, campañas publicitarias masivas, ambientes cerrados libres de humo, restricción de la publicidad), la pesquisa y la aplicación de la consejería breve ABC-D tal como se consigna en las Primeras Guías de Practica Clínica del Tratamiento del tabaquismo, Chile 2017.

Según las Tobacco Cessation Guidelines for High Risk Groups, 2017, (Guias de Cesación del Tabaco para Grupos de Alto Riesgo) realizadas en la Unión Europea ${ }^{8}$ podemos agregar que se requieren enfoques adaptados a los adolescentes para realizar intervenciones en cesación del tabaco, de los cuales deben estar en conocimiento tanto profesores y como profesionales de la salud en las guias TOB-g. Tobacco Cessation Guidelines for High Risk Population, $2017^{8}$ se mostró que los estudiantes calificaron como "efectos a largo plazo para la salud", "rendimiento deportivo deteriorado" y "disminución del atractivo" como las razones más importantes para dejar de fumar y encontró que los 
adolescentes eran indiferentes a los mensajes que han sido utilizados en múltiples campañas exitosas de prevención del tabaco juvenil que enfocan "la preocupación por no ser aceptado por sus pares "y" explotado por la industria tabacalera.

Consejos de cesación para adolescentes:

- Higiene personal, mal olor y mal aliento.

- Disminución del atractivo para el sexo opuesto.

- Reducción del rendimiento atlético.

- Enfermedad respiratoria frecuente (bronquitis, tos), problemas dentales.

- Reducción del riesgo a largo plazo de enfermedad cardiovascular, cáncer, etc.

- Costo del consumo de tabaco.

(Adaptado basado en Latimer et al. $2012^{19} \mathrm{y}$ Milton et al, 2004) ${ }^{18}$.

En relación al uso de TRN en adolescentes Las Tobacco Cessation Guidelines for High Risk Groups, 2017 recomienda fuertemente que la TRN se use en adolescentes en combinación con consejería para maximizar los resultados de la cesación (Nivel de Evidencia A) lo que coincide con la opinion de los expertos chilenos. Las Guías Australianas $2014^{9}$ consideran que la indicación de TRN en adolescentes se debe realizar con precauciones. Las Guías de Nueva Zelandia $2014^{10}$ consideran que no hay evidencia suficiente para afirmar que el uso de NRT mejora las tasas de abstinencia entre los fumadores jóvenes. No obstante, la opinión de los expertos es que la TRN puede ser considerada para el uso por los jóvenes que quieren ayuda para dejar de fumar. Las Guías Nice 2010 recomiendan la terapia de reemplazo de nicotina junto con intervenciones conductuales para pacientes con dependencia a la nicotina a partir de los 12 años $^{11}$.

Otro punto importante a tener en consideración en los niños, es la exposición al humo de segunda mano. Muchas veces los padres no tienen conciencia de los serios daños que esto ocasiona a la salud respiratoria de sus hijos o simplemente lo niegan. Al respecto los pediatras tenemos la responsabilidad de abordar el problema mediante la educación a los padres para que tomen conciencia del daño que este humo produce a sus hijos y derivarlos a cesación del tabaco que puede ser telefónica (6003607777, Salud responde MINSAL, este número aparece en los envases de cigarrillos), si es que no se dispone de otros recursos de cesación.

\section{Bibliografía}

1.- Décimo estudio nacional de drogas en población escolar de Chile, $20138^{\circ}$ básico a $4^{\circ}$ medio. Dispo- nible en: http:/www.senda.gob.cl/media/estudios/ PE/DECIMO\%20ESTUDIO\%20NACIONAL\%20 ESCOLARES_2013.pdf (Accedido en diciembre de 2016).

2.- Encuesta Mundial de Tabaquismo en Jóvenes 2016, disponible en http://web.minsal.cl/wp-content/ uploads/2017/10/EMTJ2016_17_10_2017_VF.pdf

3.- Preventing tobacco use among youth and young adults. A report of the surgeon general. Disponible en: https:// www.surgeongeneral.gov/library/reports/preventingyouth-tobacco-use/exec-summary.pdf (Consultado en diciembre de 2016).

4.- Tobacco products scientific advisory committee. Menthol cigarettes and public health: review of the scientific evidence and recommendations.. Disponible en: http://www.fda.gov/downloads/advisorycommittees/ committeesmeetingmaterials/tobaccoproductsscientificadvisorycommittee/ucm269697.pdf (Consultado en diciembre de 2016).

5.- Antecedentes técnicos que respaldan la regulación del mentol en productos del tabaco. Disponible en: http:// www.chilelibredetabaco.cl/wp-content/uploads/2015/04/ Informe-Tecnico-Prohibicion-Mentol-MINSAL.pdf (Consultado en enero de 2017).

6.- Scientific committee on emerging and newly identified health risks (SCENIHR) 2010. Addictiveness and attracktiveness of tobacco additives.Disponible en: http://ec.europa.eu/health/scientific_committees/emerging/docs/scenihr_o_029.pdf (Consultado en enero de 2017).

7.- E-cigarette use among youth and young adults. A report of the surgeon general. Executive summary 2016. Disponible en: https://e-cigarettes.surgeongeneral.gov/ documents/2016_SGR_Full_Report_non-508.pdf (Consultado en diciembre de 2016).

8.- TOB-g. Tobacco Cessation Guidelines for High Risk Population.Disponible en: http://www.tri.ie/ uploads/5/2/7/3/52736649/tob-g-book-digital-version. pdf (consultado en agosto de 2017).

9.- ZWAR N, RICHMOND R, BORLAND R, PETERS M, LITT J, BELL J, et al. Supporting Smoking Cessation:a Guide for Health Professionals. Melbourne:The Royal Australian College of General Practitioners 2011 (updated 2014).

10.- Recommendations of the New Zealand Guidelines for Helping People to Stop Smoking. Wellington: Ministry of Health 2014.

11.- National Institute for Healthcare Excellence (NICE). Smoking: stopping in pregnancy and after childbirth. Public Health Guideline [PH26] (2010). Disponible en: https://www.nice.org.uk/guidance/ph26 (Consultado el 19 de septiembre de 2017).

Correspondencia a:

Dra. Ana María Herreara G.

Email: amherrerag@yahoo.com 